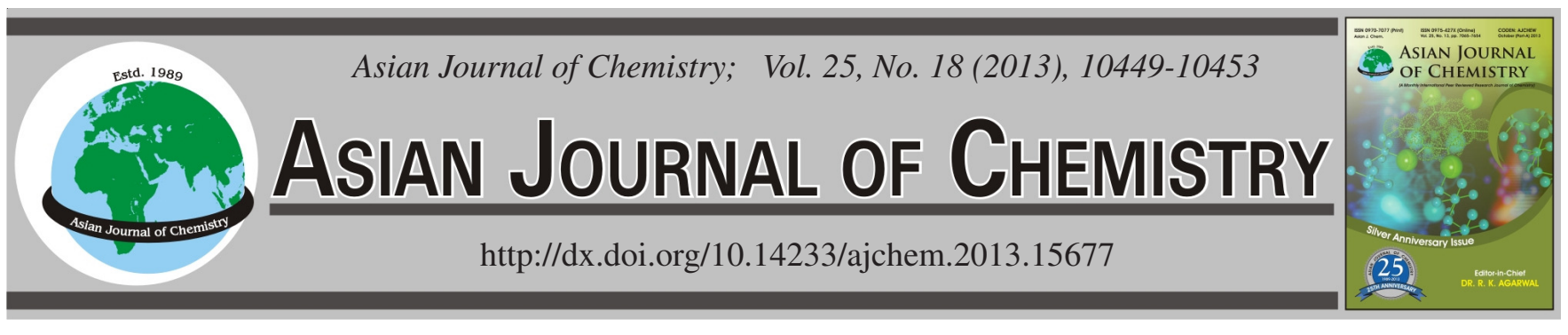

\title{
Synthesis and Evaluation of Some Novel Benzimidazole Derivatives Bearing Thiazolidinone Moiety as Potential Antimicrobial Activity
}

\author{
P. SUdHIR KUMAR ${ }^{1, *}$ and S.K. PATRO ${ }^{2}$
}

${ }^{1}$ Department of Pharmaceutical Chemistry, School of Pharmaceutical Sciences, Siksha 'O' Anusandhan University, Bhubaneswar-751 003, India ${ }^{2}$ Department of Pharmaceutical Analysis, Institute of Pharmaceutical Sciences and Technology, Salipur-754 202, India

*Corresponding author: E-mail: sairampaidesetty@gmail.com

\begin{abstract}
A new series 3-(3-hydroxy-4-(1H-benzo[d]imidazol-2-yl)phenyl)-2-substituted phenylthiazolidin-4-one (4a-h) were synthesized by the cycloaddition of 2-(benzo[d]imidazol-2-yl)-5-(4-substituted benzylideneamino)phenol (3a-h) with thioglycollic acid in the presence of anhydrous zinc chloride in 1,4 dioxane and the compounds (4a-h) further coupled with diazonium salt of sulphonilamide to give diazo substituted of thiazolidinone. The structures of these newly synthesized were characterized by elementary analysis, IR, ${ }^{1} \mathrm{H} N \mathrm{NM}$ and the synthesized compounds were evaluated in vitro for their preliminary antibacterial activity against various bacterial viz: E. coli, S. aureas, S. typhi and Pseudomonas species and of their antifungal activities versus Aspergillus niger and Aspergillus oryzae. Antibacterial and antifungal activities of each compound were compared with standards amoxicillin and fluconazole, respectively. The results revealed that some compounds were exhibited moderate to good antibacterial activity against both Gram (+) and Gram (-) bacteria whereas same compounds were exhibited moderate to weak antifungal action against both fungal strains.
\end{abstract}

Key Words: 4-Aminosalicylic acid, Thiazolidinone, Benzene-1,2-diamine, Diazotization, Antibacterial activity.

\section{INTRODUCTION}

In the past few decades, benzimidazole and its derivative have received much attention due to their chemotherapeutic values. The benzimidazole ring is an important pharmacophore in modern drug discovery. Benz[b]imidazole chemically defined as benzene fused with imidazole heterocyclic ring. Regarding structural activity relationship of benzimdazole, many literature reports have revealed that the substitution at 1,2 , and 5 position of benzimidazole is very important for enhancing antimicrobial activities. 2-(Aryl substituted) benzimidazole were exhibited wide range of pharmacological activity including antibacterial ${ }^{1}$, antiviral $^{2}$, antitumor ${ }^{3}$ and antiinflammatory ${ }^{4}$. 4-Thiazolidinone is derivatives of saturated ring of thiazole with carbonyl group at the fourth position. The chemistry and pharmacological action of 4-thiazolidinone have great interest to medicinal chemistry because of its derivative possess various biological activities such as antibacterial ${ }^{5}$, antifungal ${ }^{6}$, anticonvulsant ${ }^{7}$, antitubercular $^{8}$, anticancer ${ }^{9}$, anti-nflammatory ${ }^{10}$, antihistamine $^{11}$, antioxidant ${ }^{12}$, antihypertension ${ }^{13}$, analgesic ${ }^{14}$ and hypoglycemic activity ${ }^{15}$. As per literature survey the azo dyes have been widely used in dying textile fibers, biomedical studies, advanced applications in organic synthesis and shows variety of biological activities including antibacterial ${ }^{16,17}$ and pesticide activities $^{18}$. Synthesized azo dyes prepared by diazotization of a primary aromatic amine, followed by coupling with one or more nucleophiles. Amino, hydroxyl and active methylene groups are commonly used coupling components ${ }^{19}$. The azo dye sulphonamide of antibacterial pro-drug such as protonosil was the first effective chemotherapeutic agents that could be used systemically for the cure of bacterial infection in humans.

In the new drug design, the development of hybrid molecules through the combination of different pharmacophores in one frame may lead to compounds with interesting pharmacological properties. The co-administration of the chemical entities with insertion of sulphonamide group, acting by different mechanisms may have a synergistic effect and resulting in a higher activity than each of the components. The above mentioned both heterocyclic rings individually have been vast biological properties, which on prompted the synthesis of some privileged hybrid molecules by connecting the main structural unit of the benz[b]imidazole ring system with the 4-thiazolidinone bearing sulphonamide group and screened their antimicrobial activity.

\section{EXPERIMENTAL}

The chemicals used in the present studies were of synthetic grade, Merck company Ltd. The products were characterized by IR, ${ }^{1} \mathrm{H}$ NMR \& mass spectroscopy. The melting points were 
determined by open capillary method and uncorrected. The ${ }^{1} \mathrm{H}$ NMR spectra were measured in $\mathrm{CDCl}_{3}$ solutions on a Brucker $400 \mathrm{MHz}$ spectrometer. The Mass spectra were recorded on Shimadzu LS-MS 2010 spectrometer. The purity of prepared compounds was checked by TLC using silica gel. Benzimidazole and thiazolidinone prepared by known literature method ${ }^{20}$.

General synthesis of 5-amino-2-(benzo[d]imidazol-2yl)phenol (2): In round bottom flask, a mixture of 4-amino2-hydroxybenzoic acid ( $0.01 \mathrm{~mol})$, benzene-1,2-diamine (0.01 $\mathrm{mol})$ and polyphosphoric acid (PPA) $(15 \mathrm{~mL})$ were heated in an oil-bath at $200{ }^{\circ} \mathrm{C}$ for $3 \mathrm{~h}$. The reaction mixture was cooled and poured on to the $100 \mathrm{~mL}$ crushed ice water, neutralize with aqueous ammonia. The separated solid was filtered, washed with water and dried to obtain desired product. The crude white precipitate was purified by dissolving $10 \% \mathrm{HCl}$ and reprecipitated by addition of aqueous ammonia. The resulting solid was filtered and recrystallized from ethyl acetate. IR $(\mathrm{KBr}$, $\left.\mathrm{V}_{\max }, \mathrm{cm}^{-1}\right): 3448(\mathrm{O}-\mathrm{H}), 1530(\mathrm{C}=\mathrm{C}), 1621(\mathrm{C}=\mathrm{N}), 1278(\mathrm{C}-$ N), 894 (Ar-H).

Synthesis of 2-(benzo[d]imidazol-2-yl)-5-(4-substituted benzylideneamino)phenol (3a-h): The corresponding aromatic aldehyde $(0.01 \mathrm{~mol})$ was added to stirred solution of 5-amino-2-(1H-benzo[d]imidazol-2-yl) phenol (1) (0.01 mol) of absolute ethanol and the mixture was refluxed for $2 \mathrm{~h}$. After cooling, the reaction mixture was filtered and kept for $24 \mathrm{~h}$ and finally the crystal of desired Schiff base product of 2-(1methyl-benzo[d]imidazol-2-yl)-5-(4-substituted benzylideneamino)phenol (2a-h) recrystallized from ethanol.

2-(1H-Benzo[d]imidazol-2-yl)-5(benzylideneamino)phenol (3a): IR (KBr, $\left.v_{\max }, \mathrm{cm}^{-1}\right) ; 3030,765$ (Ar-H), (3350 (N-H), 3450 (Ar-OH), $1200(\mathrm{C}-\mathrm{O}), 1565(\mathrm{~N}=\mathrm{CH}), 1360(\mathrm{C}-\mathrm{N})$; ${ }^{1} \mathrm{H}$ NMR $(\delta) 8.56$ (s, 1H, N=CH), 9.61(s, 1H, Ar-OH), 6.89$7.86(\mathrm{~m}, 12 \mathrm{H}, \mathrm{Ar}-\mathrm{H})$.

2-(1H-Benzo[d]imidazol-2-yl)-5-(4-chlorobenzylideneamino)phenol (3b): IR ( $\left.\mathrm{KBr}, \mathrm{v}_{\max }, \mathrm{cm}^{-1}\right)$; 3035, $810(\mathrm{Ar}-\mathrm{H})$, 3350 (N-H), 3450 (Ar-OH), 1200 (C-O), 1565 (N=CH), 1403 $(\mathrm{C}-\mathrm{N}), 783$ (Ar-Cl); ${ }^{1} \mathrm{H}$ NMR $(\delta) 8.56$ (s, 1H, N=CH), 9.61 (s, $1 \mathrm{H}, \mathrm{Ar}-\mathrm{OH}), 6.89-7.77$ (m, 11H, Ar-H).

2-(1H-Benzo[d]imidazol-2-yl)-5-(4-methoxybenzylideneamino)phenol (3c): IR ( $\left.\mathrm{KBr}, \mathrm{v}_{\max }, \mathrm{cm}^{-1}\right)$; 3050, 815 (Ar$\mathrm{H}), 3350(\mathrm{~N}-\mathrm{H}), 3455$ (Ar-OH), $1200(\mathrm{C}-\mathrm{O}), 1560(\mathrm{~N}=\mathrm{CH})$, $1375(\mathrm{C}-\mathrm{N}), 1250\left(\mathrm{Ar}^{-} \mathrm{OCH}_{3}\right) ;{ }^{1} \mathrm{H}$ NMR $(\delta) 8.56(\mathrm{~s}, 1 \mathrm{H}$, $\mathrm{N}=\mathrm{CH}), 9.61(\mathrm{~s}, 1 \mathrm{H}, \mathrm{Ar}-\mathrm{OH}), 6.89-7.86(\mathrm{~m}, 11 \mathrm{H}, \mathrm{Ar}-\mathrm{H}) 3.83(\mathrm{~s}$, $\left.3 \mathrm{H} \mathrm{Ar}-\mathrm{OCH}_{3}\right)$.

2-(1H-Benzo[d]imidazol-2-yl)-5-(4-nitrobenzylideneamino)phenol (3d) : IR ( $\left.\mathrm{KBr}, \mathrm{v}_{\max }, \mathrm{cm}^{-1}\right)$; 3050, $815(\mathrm{Ar}-\mathrm{H})$, 3350 (N-H), 3350 (Ar-OH), 1200 (C-O), 1560 (N=CH), 1380 $(\mathrm{C}-\mathrm{N}), 1520,1345\left(\mathrm{Ar}-\mathrm{NO}_{2}\right) ;{ }^{1} \mathrm{H}$ NMR $(\delta) 8.56(\mathrm{~s}, 1 \mathrm{H}, \mathrm{N}=\mathrm{CH})$, 9.61(s, 1H, Ar-OH), 6.89-7.86 (m, 11H, Ar-H) .

2-(1H-Benzo[d]imidazol-2-yl)-5-(2-methylbenzylideneamino)phenol (3e) : IR ( $\left.\mathrm{KBr}, \mathrm{V}_{\max }, \mathrm{cm}^{-1}\right) ; 2985\left(\mathrm{Ar}-\mathrm{CH}_{3}\right)$, 3050, 735 (Ar-H), 3450 (N-H), 3350 (Ar-OH), 1200 (C-O), $1560(\mathrm{~N}=\mathrm{CH}) ;{ }^{1} \mathrm{H}$ NMR $(\delta) 8.56(\mathrm{~s}, 1 \mathrm{H}, \mathrm{N}=\mathrm{CH}), 9.61(\mathrm{~s}, 1 \mathrm{H}$, Ar-OH), 6.89-7.86 (m, 11H, Ar-H) 2.83 (s, 3H, $\mathrm{CH}_{3}$ ).

5-[(4-(1H-Benzo[d]imidazol-2-yl)-3-hydroxyphenylimino)methyl]-2-methoxyphenol (3f): $\mathrm{IR}\left(\mathrm{KBr}, \mathrm{v}_{\max }, \mathrm{cm}^{-1}\right)$; 3050,855 (Ar-H), 3350 (N-H), 3460 (Ar-OH), 1200 (C-O),
$1235\left(\mathrm{Ar}-\mathrm{OCH}_{3}\right) 1560(\mathrm{~N}=\mathrm{CH}) ;{ }^{1} \mathrm{H}$ NMR $(\delta) 8.56(\mathrm{~s}, 1 \mathrm{H}$, $\mathrm{N}=\mathrm{CH}), 9.61(\mathrm{~s}, 1 \mathrm{H}, \mathrm{Ar}-\mathrm{OH}), 6.89-7.86(\mathrm{~m}, 11 \mathrm{H}, \mathrm{Ar}-\mathrm{H}) 3.83$ $\left(\mathrm{s}, 3 \mathrm{H}, \mathrm{OCH}_{3}\right)$.

Synthesis of 2-aryl substituted thiazolidin-4-ones (4a-h): A mixture of Schiff base $(0.001 \mathrm{~mol})$ and thioglycollic acid $(0.001 \mathrm{~mol})$ dissolved in 1,4 dioxane $(20 \mathrm{~mL})$, a pinch of anhydrous zinc chloride $(0.5 \mathrm{mg})$ was added and refluxed for $8 \mathrm{~h}$. The reaction was then cooled and the resulting solid was washed with sodium bicarbonate solution and final compound 3-[4-(1H-benzo[d]imidazol-2-yl)-3-hydroxyphenyl]-2-aryl substituted thiazolidin-4-one (4a-f) recrystallized from absolute ethanol.

3-[4-(1H-Benzo[d]imidazol-2-yl)-3-hydroxyphenyl]-2phenylthiazolidin-4-one (4a): IR ( $\left.\mathrm{KBr}, \mathrm{v}_{\max }, \mathrm{cm}^{-1}\right)$; $3030(\mathrm{Ar}-\mathrm{H})$, 3350 (N-H), 3460 (Ar-OH), 1700 (C=O), 1405 (C-N), 1610 $(\mathrm{C}=\mathrm{N}), 1120(\mathrm{C}-\mathrm{S}) ;{ }^{1} \mathrm{H}$ NMR $(\delta)$ 6.86-7.58 (m, 12H, Ar-H), 9.61 (s, 1H, Ar-OH), 12.56 (s, 1H,-NH-benzimidazole), 6.44 (s, 1H, CH-thiazolidinone), 3.9 (s, 2H, $\mathrm{CH}_{2}$-thiazolidin-one); $\mathrm{m} / \mathrm{z}: 387.10(100.0 \%), 388.11(24.1 \%)$.

3-[4-(1H-Benzo[d]imidazol-2-yl)-3-hydroxyphenyl]-2(4-chlorophenyl)thiazolidin-4-one (4b): IR ( $\left.\mathrm{KBr}, \mathrm{v}_{\max }, \mathrm{cm}^{-1}\right)$; 3021 (Ar-H), 3350 (N-H), 3450 (Ar-OH), 1403 (C-N), 1610 $(\mathrm{C}=\mathrm{N}), 783(\mathrm{Ar}-\mathrm{Cl}), 1695(\mathrm{C}=\mathrm{O}), 1118(\mathrm{C}-\mathrm{S}) ;{ }^{1} \mathrm{H}$ NMR $(\delta)$; 7.16-7.67 (m, 11H, Ar-H), 9.61 (s, 1H, Ar-OH), 12.56 (s, 1H, -NH-benzimidazole), 6.44 (s, 1H, CH-thiazolidinone), 4.0 (s, 2H, $\mathrm{CH}_{2}$-thiazolidinone); $\mathrm{m} / \mathrm{z}: 421.07$ (100.0\%), 423.06 $(36.5 \%)$.

3-[4-(1H-Benzo[d]imidazol-2-yl)-3-hydroxyphenyl]-2(4-methoxyphenyl)thiazolidin-4-one (4c): IR ( $\left.\mathrm{KBr}, \mathrm{v}_{\max }, \mathrm{cm}^{-1}\right)$; 3011 (Ar-H), $3390(\mathrm{~N}-\mathrm{H}), 1420(\mathrm{C}-\mathrm{N}), 1602(\mathrm{C}=\mathrm{N}), 1214$ $\left(\mathrm{Ar}-\mathrm{OCH}_{3}\right), 1684(\mathrm{C}=\mathrm{O}), 1109$ (C-S); ${ }^{1} \mathrm{H}$ NMR $(\delta) ; 6.86-7.84$ (m, 11H, Ar-H), 9.61 (s, 1H, Ar-OH), 3.83 (s, 3H, Ar- $-\mathrm{OCH}_{3}$ ), 12.56 (s, 1H,-NH-benzimidazole), 6.44 (s, 1H, CH-thiazolidinone), 3.9 (s, $2 \mathrm{H}, \mathrm{CH}_{2}$-thiazolidinone); $\mathrm{m} / \mathrm{z}: 417.11$ (100\%), $418.12(25.2 \%)$.

3-[4-(1H-Benzo[d]imidazol-2-yl)-3-hydroxyphenyl]-2(4-nitrophenyl)thiazolidin-4-one (4d): IR $\left(\mathrm{KBr}, \mathrm{v}_{\max }, \mathrm{cm}^{-1}\right)$; 3077 (Ar-H), 3340 (N-H), 3450 (Ar-OH), 1417 (C-N), 1598 $(\mathrm{C}=\mathrm{N}), 1515,1343\left(\mathrm{Ar}-\mathrm{NO}_{2}\right), 1701(\mathrm{C}=\mathrm{O}), 1105(\mathrm{C}-\mathrm{S}) ;{ }^{1} \mathrm{H}$ NMR ( $\delta$ ); 6.86-7.58 (m, 11H, Ar-H), 9.61(s, 1H, Ar-OH), 12.56 (s, 1H,-NH-benzimidazole), 6.44(s,1H,CH-thiazolid-inone), 4.0 (s, 2H, $\mathrm{CH}_{2}$-thiazolidinone); $\mathrm{m} / \mathrm{z}: 432.09$ (100.0\%), 433.09 $(26.2 \%)$.

3-[4-(1H-Benzo[d]imidazol-2-yl)-3-hydroxyphenyl]-2o-tolylthiazolidin-4-one (4e): IR (KBr, $\left.v_{\max }, \mathrm{cm}^{-1}\right)$; 3023 (Ar$\mathrm{H}), 3359$ (N-H), 3460 (Ar-OH), $1393(\mathrm{C}-\mathrm{N}), 1604(\mathrm{C}=\mathrm{N})$, 2919, $2857\left(\mathrm{Ar}^{-\mathrm{CH}_{3}}\right), 1700(\mathrm{C}=\mathrm{O}), 1117(\mathrm{C}-\mathrm{S}) ;{ }^{1} \mathrm{H}$ NMR $(\delta)$; 6.86-7.58 (m,11H, Ar-H), 9.61(s,1H, Ar-OH), 12.56 (s, 1H,NH-benzimidazole), 2.83(s, 3H, Ar- $\left.\mathrm{CH}_{3}\right), 6.44(\mathrm{~s}, 1 \mathrm{H}, \mathrm{CH}-$ thiazolidinone), 4.0 (s, $2 \mathrm{H}, \mathrm{CH}_{2}$-thiazolidinone).

3-[4-(1H-Benzo[d]imidazol-2-yl)-3-hydroxyphenyl]-2(3-hydroxy-4-methoxyphenyl)thiazolidin-4-one (4f): IR $\left(\mathrm{KBr}, v_{\max }, \mathrm{cm}^{-1}\right) ; 3033(\mathrm{Ar}-\mathrm{H}), 3324(\mathrm{~N}-\mathrm{H}), 3455$ (Ar-OH), $1394(\mathrm{C}-\mathrm{N}), 1604(\mathrm{C}=\mathrm{N}), 1208\left(\mathrm{Ar}-\mathrm{OCH}_{3}\right), 3356(\mathrm{Ar}-\mathrm{OH})$, 1719 (C=O), 1108 (C-S); ${ }^{1} \mathrm{H}$ NMR $(\delta) ; 6.86-7.58$ (m, 10H, Ar-H), 9.61 (s, 1H, Ar-OH), 3.83 (s, 3H, Ar- $\mathrm{OCH}_{3}$ ), 12.56 (s, 1H, -NH-benzimidazole), 6.44 (s, 1H, CH-thiazolidinone), 4.0 (s, 2H, $\mathrm{CH}_{2}$-thiazolidinone). 
3-[4-(1H-Benzo[d]imidazol-2-yl)-3-hydroxyphenyl]-2[4-(dimethylamino)phenyl]thiazolidin-4-one (4g): IR ( $\mathrm{KBr}$, $\left.v_{\max }, \mathrm{cm}^{-1}\right) ; 3033(\mathrm{Ar}-\mathrm{H}), 3324(\mathrm{NH}), 3460(\mathrm{Ar}-\mathrm{OH}), 1719$ $(\mathrm{C}=\mathrm{O}), 1108(\mathrm{C}-\mathrm{S}), 2790,2730\left(\mathrm{Ar}-\mathrm{N}\left(\mathrm{CH}_{3}\right)_{2}\right) ;{ }^{1} \mathrm{H}$ NMR $(\delta)$; 6.86-7.84 (m, 11H, Ar-H), $9.61(\mathrm{~s}, 1 \mathrm{H}, \mathrm{Ar}-\mathrm{OH}), 3.03$ (s, 6H, Ar-N $\left.\left(\mathrm{CH}_{3}\right)_{2}\right), 12.56$ (s, 1H,-NH-benzimidazole), 6.44 (s, 1H, $\mathrm{CH}$-thiazolidinone), 4.0 (s, 2H, $\mathrm{CH}_{2}$-thiazolidinone).

3-[4-(1H-Benzo[d]imidazol-2-yl)-3-hydroxyphenyl]-2(furan-2-yl)thiazolidin-4-one (4h): IR ( $\left.\mathrm{KBr}, \mathrm{v}_{\max }, \mathrm{cm}^{-1}\right) ; 3150$ (furanyl-H), $3324(\mathrm{~N}-\mathrm{H}), 3460(\mathrm{Ar}-\mathrm{OH}), 1715(\mathrm{C}=\mathrm{O}), 1110$ $(\mathrm{C}-\mathrm{S}) ;{ }^{1} \mathrm{H}$ NMR $(\delta)$; 6.89-7.67 (m, 7H, Ar-H), 9.61 (s, 1H, Ar$\mathrm{OH}), 12.56$ (s, 1H,-NH-benzimidazole), 6.26-7.67 (m, 3H, furanyl-H) 6.5 (s, 1H, CH-thiazolidinone), $3.9\left(\mathrm{~s}, 2 \mathrm{H}, \mathrm{CH}_{2}-\right.$ thiazolidinone).

Synthesis of 4 -[(3-(1H-benzo[d]imidazol-2-yl)-2hydroxy-6-(4-oxo-2-substituitedphenylthiazolidin-3-yl)phenyl) diazenyl]benzene sulfonamide (5a-h): A cold solution of $2.5 \mathrm{~mL}$ of sodium nitrite was added drop wise to ice cold solution of sulphanilamide in conc. $\mathrm{HCl}$ and water. The temperature of the reaction was maintained up to $0-5{ }^{\circ} \mathrm{C}$ during addition. When addition was completed, the solution was kept for 5 min. with occasional stirring to complete the diazotization. Then, it was poured into an ice cold solution of 2-aryl substituted thiazolidin-4-one in $10 \%(20 \mathrm{~mL})$ sodium hydroxide solution. The reaction mixture was allowed to stand in an ice bath for 10-15 min. The coloured products obtained were filtered, washed with water and finally dried. The entire product individually was recrystallized from $50 \%$ ethanol.

4-[(3-(1H-Benzo[d]imidazol-2-yl)-2-hydroxy-6-(4-oxo2-phenylthiazolidin-3-yl)phenyl)diazenyl]benzene sulfonamide (5a): IR (KBr, $\left.v_{\max }, \mathrm{cm}^{-1}\right)$; $3030(\mathrm{Ar}-\mathrm{H}), 3350(\mathrm{~N}-\mathrm{H})$, 3460 (Ar-OH), $1700(\mathrm{C}=\mathrm{O}), 1405(\mathrm{C}-\mathrm{N}), 1610(\mathrm{C}=\mathrm{N}), 1120$ $(\mathrm{C}-\mathrm{S}), 1556(\mathrm{~N}=\mathrm{N}), 1317,1147\left(\mathrm{SO}_{2}\right) ;{ }^{1} \mathrm{H} \mathrm{NMR}(\delta)$ 6.86-7.58 (m, 11H, Ar-H), 9.82 (s, 1H, Ar-OH), 12.56 (s, 1H, -NH-benzimidazole), 6.44 (s, $1 \mathrm{H}, \mathrm{CH}$-thiazolidinone), 4.0 (s, 2H, $\mathrm{CH}_{2}-$ thiazolidinone), 7.25 (s, $2 \mathrm{H}, \mathrm{NH}_{2}$ of sulphonamide), 8.1-8.25 (m, 4H, Aryl azo sulphonamide).

4-[(3-(1H-Benzo[d]imidazol-2-yl)-2-hydroxy-6-(4-oxo2-chlorophenylthiazolidin-3-yl) phenyl)diazenyl]benzene sulfonamide (5b): IR (KBr, $\left.v_{\max }, \mathrm{cm}^{-1}\right) ; 3030(\mathrm{Ar}-\mathrm{H}), 3350$ $(\mathrm{N}-\mathrm{H}), 3460(\mathrm{Ar}-\mathrm{OH}), 1700(\mathrm{C}=\mathrm{O}), 1405(\mathrm{C}-\mathrm{N}), 1610(\mathrm{C}=\mathrm{N})$, $1120(\mathrm{C}-\mathrm{S}), 1556(\mathrm{~N}=\mathrm{N}), 1317,1147\left(\mathrm{SO}_{2}\right) ;{ }^{1} \mathrm{H}$ NMR $(\delta) 6.86-$ 7.58 (m, 10H, Ar-H), 9.82 (s, 1H, Ar-OH), 12.56 (s, 1H, -NHbenzimidazole), 6.44 (s, 1H, CH-thiazolidinone), 4.0 (s, 2H, $\mathrm{CH}_{2}$-thiazolidinone), 7.25 (s $2 \mathrm{H}, \mathrm{NH}_{2}$ of sulphonamide), 8.18.25 (m, 4H, arylazosulphonamide), $\mathrm{m} / z$ : 604.08 (100.0\%).

Antimicrobial activities: All the synthesized compounds (3-5) were evaluated for their antibacterial activity against Escherichia coli (MTCC-723), Staphylococcus aureus (ATCC29513), Pseudomonas aeruginosa (MTCC-1688) Salmonella typhi (recultred) and of their antifungal activity against Aspergillus niger (MTCC-281), Aspergillus oryzae (recultred) by the cup-plate method. Holes of $6 \mathrm{~mm}$ diameter were punched carefully using a sterile cork borer and these were filled with test solution different concentrations 1000, 750 and $500 \mu \mathrm{g} / \mathrm{mL}$. The plates were incubated at $37{ }^{\circ} \mathrm{C}$ for $24 \mathrm{~h}$ and $72 \mathrm{~h}$ in case of antibacterial and antifungal activity, respectively. The diameter of the zone of inhibition $(\mathrm{mm})$ was measured for all the test compounds and results were compared with the standard drugs amoxicillin and fluconazole for antibacterial and antifungal activity, respectively.

\section{RESULTS AND DISCUSSION}

The starting material 2-substituted benzimidazole (2) was prepared according to a reported procedure through the reaction of benzene-1,2-diamine with appropriate $p$-amino salicylic $\operatorname{acid}^{22}$. The compound $\mathbf{2}$ further on condensation with various selected aromatic aldehydes furnished Schiff bases, 2'-(benzo[d]imidazol-2-yl)-5'-(4-substituted benzylideneamino) phenol (3a-h). The 5-membered heterocyclic ring such as thiazolidinone in compound 4 was incorporated by the cycloaddition of compounds (3a-h) with thioglycollic acid in the presence of anhydrous aluminum chloride as a catalyst to give 3-(3-hydroxy-4-(1Hbenzo[d]imidazol-2-yl)phenyl)-2-substituted phenylthiazolidin4-one (4a-h). The cycloaddition mechanism of reaction was mentioned in Scheme-I. The azo sulphonamide group in compound $\mathbf{5}$ was introduced by coupling with diazonium salt of sulphanilamide furnished products $4-((3-(1 H$-benzo[d]imidazol-2-yl)-2-hydroxy-6-(4-oxo-2-substituited phenylthiazolidin-3-yl)phenyl)diazenyl)benzene sulfonamide (5a-h). Finally the products were recrystallized with glacial acetic acid and water in (1:3). The purity was checked by the preparative TLC using silica Gel and a structure of the individual products were characterized by $\mathrm{C}, \mathrm{H}, \mathrm{N}$ analysis, I R and ${ }^{1} \mathrm{NMR}$ spectral data. The physical data and the percentage yield of the synthesized compounds are given in Table- 1 .

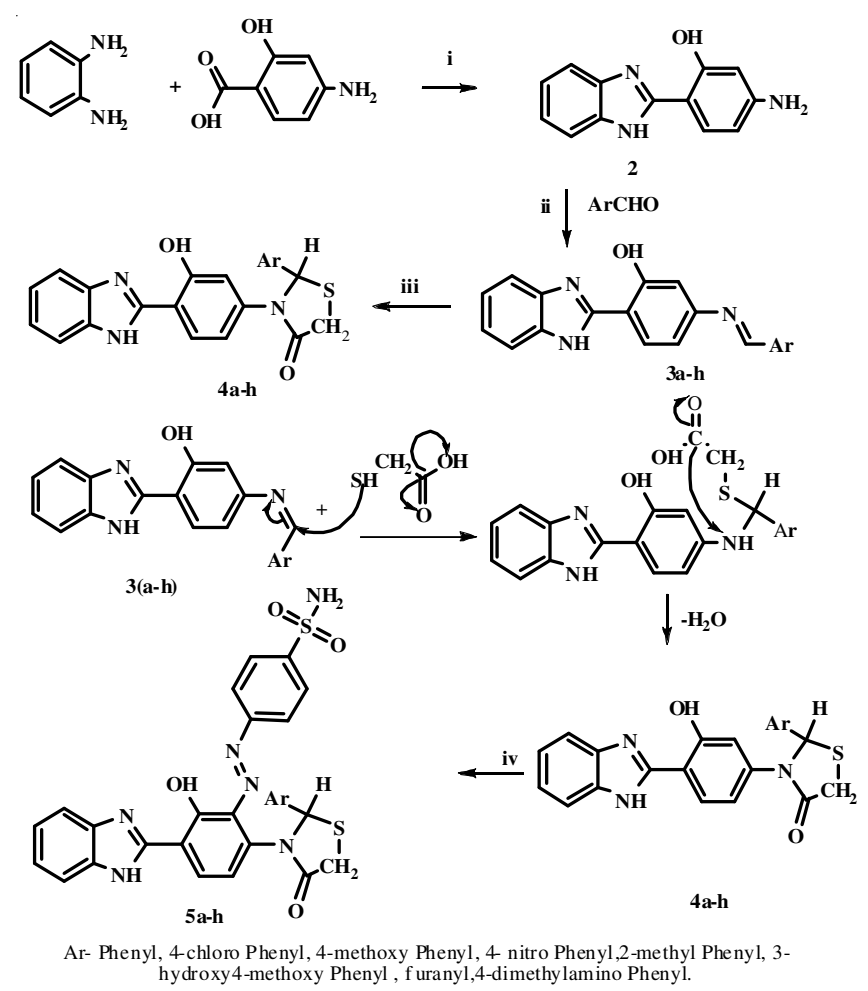

Scheme-I: Reagents: (i) polyphosphuric acid, $3 \mathrm{~h}, 86 \%$; (ii) ethanol, 2h, 75 $\%$; (iii) thioglycolic acid in anhydrous zinc chloride (iv) sodium nitrite, $\mathrm{HCl} / 0-5{ }^{\circ} \mathrm{C}$

The following peaks confirmed the formation of thiazolidinone. The peaks at 1720-1715, 1410-1398 and 11201110 in FTIR $\left(\mathrm{cm}^{-1}\right)$ have shown the groups of $\mathrm{C}=\mathrm{O}, \mathrm{C}-\mathrm{N}, \mathrm{C}-\mathrm{S}$ of thiazolidinone respectively. The ${ }^{1} \mathrm{H}$ proton of imidazole is 
TABLE-1

PHYSICAL DATA OF SYNTHESIZED COMPOUNDS 3(a-h) AND 4(a-h)

\begin{tabular}{|c|c|c|c|c|c|c|}
\hline \multirow{2}{*}{ Compounds } & \multirow{2}{*}{ Aryl } & \multirow{2}{*}{$\begin{array}{c}\text { m.f. } \\
\text { (m.w.) }\end{array}$} & \multicolumn{3}{|c|}{ Elemental analysis (\%): Found (calculated) } & \multirow{2}{*}{ Yield $(\%)$} \\
\hline & & & $\mathrm{C}$ & $\mathrm{H}$ & $\mathrm{N}$ & \\
\hline $3 \mathbf{3 a}$ & Phenyl- & $\mathrm{C}_{20} \mathrm{H}_{15} \mathrm{~N}_{3} \mathrm{O}(311)$ & $76.66(76.62)$ & $4.82(4.75)$ & $13.52(13.50)$ & 75 \\
\hline $3 \mathbf{b}$ & 4-Chloro phenyl & $\mathrm{C}_{20} \mathrm{H}_{14} \mathrm{~N}_{3} \mathrm{OCl}(347)$ & $64.86(64.85)$ & $4.06(4.00)$ & $12.08(12.00)$ & 65 \\
\hline $3 c$ & 4-Methoxy phenyl & $\mathrm{C}_{21} \mathrm{H}_{17} \mathrm{~N}_{3} \mathrm{O}_{2}$ & $73.45(73.35)$ & $4.99(4.88)$ & $12.24(12.20)$ & 80 \\
\hline 3d & 4-Nitro phenyl & $\mathrm{C}_{20} \mathrm{H}_{14} \mathrm{~N}_{4} \mathrm{O}_{3}(358)$ & $67.03(66.08)$ & $3.94(3.90)$ & $15.63(15.50)$ & 75 \\
\hline $3 e$ & 4-Methyl phenyl & $\mathrm{C}_{21} \mathrm{H}_{17} \mathrm{~N}_{3} \mathrm{O}(327)$ & $77.04(76.82)$ & $5.84(5.55)$ & $12.83(9.90)$ & 85 \\
\hline $3 f$ & 3-Hydroxy 4-methoxy phenyl & $\mathrm{C}_{21} \mathrm{H}_{17} \mathrm{~N}_{3} \mathrm{O}_{3}$ (359) & $70.18(70.15)$ & $4.77(4.72)$ & $11.69(11.60)$ & 45 \\
\hline $3 g$ & Furanyl- & $\mathrm{C}_{18} \mathrm{H}_{13} \mathrm{~N}_{3} \mathrm{O}_{2}(303)$ & $71.28(71.15)$ & $2.91(2.90)$ & $13.50(13.48)$ & 85 \\
\hline $3 h$ & 4-Dimethyl amino & $\mathrm{C}_{22} \mathrm{H}_{20} \mathrm{~N}_{4} \mathrm{O}(356)$ & $74.14(74.10)$ & $5.66(5.60)$ & $15.17(15.15)$ & 80 \\
\hline $4 a$ & Phenyl & $\mathrm{C}_{22} \mathrm{H}_{17} \mathrm{~N}_{3} \mathrm{O}_{2} \mathrm{~S}$ (387) & $68.20(68.15)$ & $4.42(4.38)$ & $10.85(10.75)$ & 60 \\
\hline $4 b$ & 4-Chloro phenyl & $\mathrm{C}_{22} \mathrm{H}_{16} \mathrm{~N}_{3} \mathrm{O}_{2} \mathrm{SCl}(421)$ & $62.63(62.53)$ & $3.86(3.80)$ & $9.96(9.83)$ & 85 \\
\hline $4 c$ & 4-Methoxy phenyl & $\mathrm{C}_{23} \mathrm{H}_{19} \mathrm{~N}_{3} \mathrm{O}_{3} \mathrm{~S}(417)$ & $66.17(66.10)$ & $4.59(4.55)$ & $10.08(10.00)$ & 75 \\
\hline 4d & 4-Nitro phenyl & $\mathrm{C}_{22} \mathrm{H}_{16} \mathrm{~N}_{4} \mathrm{O}_{4} \mathrm{~S}$ (432) & $61.10(61.05)$ & $3.73(3.63)$ & $14.80(14.75)$ & 60 \\
\hline $4 e$ & 4-Methyl phenyl & $\mathrm{C}_{23} \mathrm{H}_{19} \mathrm{~N}_{3} \mathrm{O}_{2} \mathrm{~S}(401)$ & $68.81(68.75)$ & $4.77(4.67)$ & $10.47(10.40)$ & 55 \\
\hline $4 f$ & 3-Hydroxy 4-methoxy phenyl & $\mathrm{C}_{23} \mathrm{H}_{19} \mathrm{~N}_{3} \mathrm{O}_{4} \mathrm{~S}(433)$ & $63.73(63.6)$ & $4.42(4.35)$ & $9.69(9.65)$ & 60 \\
\hline $4 \mathrm{~g}$ & Furanyl- & $\mathrm{C}_{20} \mathrm{H}_{15} \mathrm{~N}_{3} \mathrm{O}_{3} \mathrm{~S}$ (373) & $63.65(63.55)$ & $4.01(3.90)$ & $11.13(11.05)$ & 45 \\
\hline $4 \mathrm{~h}$ & 4-Dimethyl amino phenyl & $\mathrm{C}_{24} \mathrm{H}_{22} \mathrm{~N}_{4} \mathrm{O}_{2} \mathrm{~S}(430)$ & $66.96(66.80)$ & $5.15(5.10)$ & $13.01(12.90)$ & 50 \\
\hline $5 \mathbf{a}$ & Phenyl & $\mathrm{C}_{28} \mathrm{H}_{22} \mathrm{~N}_{6} \mathrm{O}_{4} \mathrm{~S}_{2}(570)$ & $58.93(58.85)$ & $3.86(3.75)$ & $14.63(14.55)$ & 75 \\
\hline $5 \mathbf{b}$ & 4-Chloro phenyl & $\mathrm{C}_{28} \mathrm{H}_{21} \mathrm{~N}_{6} \mathrm{O}_{4} \mathrm{~S}_{2} \mathrm{Cl}(605$ & $55.58(55.50)$ & $3.50(3.45)$ & $13.89(13.84)$ & - \\
\hline $5 c$ & 4-Methoxy phenyl & $\mathrm{C}_{29} \mathrm{H}_{24} \mathrm{~N}_{6} \mathrm{O}_{5} \mathrm{~S}_{2}(600)$ & $57.99(57.85)$ & $4.03(3.85)$ & $13.99(13.85)$ & - \\
\hline 5d & 4-Nitro phenyl & $\mathrm{C}_{28} \mathrm{H}_{21} \mathrm{~N}_{7} \mathrm{O}_{6} \mathrm{~S}_{2}$ & $54.63(54.55)$ & $3.44(3.33)$ & $15.93(15.86)$ & - \\
\hline
\end{tabular}

observed as a singlet at $12.56 \mathrm{ppm}$ apparently due to deshielding caused by the benz[b]imidazole ring. The infrared spectrum of compounds 3a-h indicated the absence of the $\mathrm{NH}_{2}$ absorption band and contains characteristic absorption band at $1685-$ $1630 \mathrm{~cm}^{-1}$, a typical of the stretching vibration of the carbonnitrogen double bond and evidently the formation of Schiff base, which further confirmed the presence of $\delta 8.56 \mathrm{ppm}$ with respect to $-\mathrm{N}=\mathrm{CH}$, interpreted by NMR. The ${ }^{1} \mathrm{H}$ NMR spectrum of compounds $\mathbf{4 a - h}$ revealed the presence of a singlet at $\delta 4.0 \mathrm{ppm}$ with respect to $\mathrm{CH}_{2}$ moiety of thiazolidinone. IR spectrum of compounds $\mathbf{5}$ a-f revealed the presence of characteristic absorption bands at 1317, $1149 \mathrm{~cm}^{-1}$ and 3200, 3260

TABLE-2

ANTIBACTERIAL PROPERTIES OF THE SYNTHESIZED COMPOUNDS

\begin{tabular}{|c|c|c|c|c|c|c|c|c|c|c|c|c|}
\hline \multicolumn{13}{|c|}{ Concentration $(\mu \mathrm{g} / \mathrm{mL})$ (zone of inhibition in $\mathrm{mm})$} \\
\hline \multirow{2}{*}{ Compound } & \multicolumn{3}{|c|}{ Escherichia coli } & \multicolumn{3}{|c|}{ Staphylococcus aureus } & \multicolumn{3}{|c|}{ Pseudomonas aeruginosa } & \multicolumn{3}{|c|}{ Salmonella typhi } \\
\hline & 1000 & 750 & 500 & 1000 & 750 & 500 & 1000 & 750 & 500 & 1000 & 750 & 500 \\
\hline $\mathbf{3 a}$ & 9 & 8 & 7 & 9 & 8 & 6 & 8 & 8 & 7 & 5 & 3 & 2 \\
\hline $\mathbf{3 b}$ & 12 & 11 & 10 & 12 & 11 & 10 & 6 & 5 & 5 & 5 & 2 & 2 \\
\hline $3 c$ & 10 & 10 & 9 & 11 & 11 & 10 & 9 & 9 & 8 & NA & NA & NA \\
\hline 3d & 12 & 12 & 11 & 14 & 13 & 11 & 10 & 9 & 8 & 4 & 3 & 2 \\
\hline $3 e$ & 10 & 9 & 8 & 9 & 8 & 7 & 7 & 6 & 5 & NA & NA & NA \\
\hline 3f & 5 & 5 & 4 & 7 & 6 & 6 & 7 & 7 & 5 & NA & NA & NA \\
\hline $3 g$ & 8 & 7 & 6 & 8 & 7 & 6 & 7 & 6 & 5 & NA & NA & NA \\
\hline $3 h$ & 7 & 6 & 5 & 8 & 7 & 6 & 7 & 6 & 5 & 3 & 2 & 2 \\
\hline $4 a$ & 10 & 9 & 8 & 10 & 9 & 7 & 7 & 7 & 6 & 4 & 3 & 2 \\
\hline $4 b$ & 12 & 11 & 11 & 14 & 13 & 12 & 12 & 11 & 10 & 6 & 5 & 5 \\
\hline $4 c$ & 9 & 8 & 7 & 10 & 8 & 7 & 7 & 6 & 5 & 5 & 3 & 2 \\
\hline 4d & 13 & 12 & 11 & 15 & 14 & 12 & 9 & 8 & 7 & 3 & 2 & 2 \\
\hline $4 f$ & 9 & 8 & 8 & 10 & 9 & 8 & 7 & 6 & 5 & 4 & 4 & 3 \\
\hline $4 \mathrm{~g}$ & 5 & 5 & 4 & 6 & 5 & 4 & 7 & 6 & 5 & 5 & 4 & 4 \\
\hline 4h & 6 & 6 & 5 & 6 & 5 & 4 & 7 & 6 & 5 & 3 & 2 & 1 \\
\hline $5 \mathbf{a}$ & 9 & 8 & 6 & 9 & 8 & 7 & 7 & 6 & 5 & 2 & 2 & 1 \\
\hline $5 b$ & 14 & 13 & 12 & 14 & 13 & 12 & 13 & 12 & 11 & 5 & 4 & 3 \\
\hline $5 c$ & 10 & 10 & 9 & 10 & 9 & 8 & 8 & 7 & 6 & 3 & 3 & 2 \\
\hline $5 d$ & 13 & 12 & 11 & 14 & 13 & 12 & 8 & 7 & 7 & 5 & 4 & 2 \\
\hline $5 e$ & 6 & 5 & 5 & 7 & 6 & 5 & 7 & 6 & 5 & 3 & 2 & 1 \\
\hline $5 f$ & 6 & 5 & 4 & 7 & 6 & 5 & 6 & 5 & 4 & 3 & 2 & 1 \\
\hline $5 g$ & 5 & 4 & 3 & 6 & 5 & 4 & 7 & 5 & 4 & NA & NA & NA \\
\hline $5 h$ & 6 & 5 & 4 & 7 & 6 & 5 & 6 & 5 & 4 & NA & NA & NA \\
\hline Std. & 17 & 16 & 15 & 18 & 17 & 16 & 15 & 14 & 13 & 15 & 15 & 14 \\
\hline
\end{tabular}

Std. Amoxicillin; NA; no activity. 
$\mathrm{cm}^{-1}$ for $\mathrm{SO}_{2}$ and $\mathrm{N}-\mathrm{H}$ str. of sulphonamide, respectively. Compounds 5a-h showed N=N additional IR peak at 1560$1550 \mathrm{~cm}^{-1}$, which confirmed the formation of azosulphonamide. The phenolic -OH group of entire compounds were chemically detected by the treatment with $\mathrm{FeCl}_{3}$ solution, which gives characteristic colour.

Antimicrobial activities: The synthesized derivatives were screened for their in vitro antibacterial and antifungal activities against $P$. aeruginosa, $S$. aureus, $S$. typhi, E. coli, Aspergillus niger and Aspergillus oryzae using ampicillin and fluconazole as a reference standard drugs. The results of both antibacterial and antifungal activity screening of the tested compounds were summarized in Tables 2 and 3.

\begin{tabular}{|c|c|c|c|c|c|c|}
\hline \multicolumn{7}{|c|}{$\begin{array}{c}\text { TABLE-3 } \\
\text { ANTIFUNGAL PROPERTIES OF THE } \\
\text { SYNTHESIZED COMPOUNDS }\end{array}$} \\
\hline \multicolumn{7}{|c|}{ Conc. $(\mu \mathrm{g} / \mathrm{mL})$ (zone of inhibition in $\mathrm{mm})$} \\
\hline \multirow{2}{*}{ Compound } & \multicolumn{3}{|c|}{ Aspergillus niger } & \multicolumn{3}{|c|}{ Aspergillus oryzae } \\
\hline & 1000 & 750 & 500 & 1000 & 750 & 500 \\
\hline $4 \mathbf{4 a}$ & 7 & 6 & 5 & 8 & 7 & 6 \\
\hline 4b & 10 & 10 & 9 & 11 & 10 & 10 \\
\hline $4 c$ & 5 & 4 & 3 & 8 & 7 & 5 \\
\hline 4d & 6 & 6 & 5 & 5 & 5 & 4 \\
\hline $4 e$ & 6 & 6 & 5 & 4 & 3 & 1 \\
\hline $4 f$ & 7 & 6 & 5 & 4 & 3 & 3 \\
\hline $4 g$ & 5 & 4 & 3 & 4 & 3 & 2 \\
\hline $4 h$ & 4 & 3 & 2 & 5 & 4 & 4 \\
\hline $5 \mathbf{a}$ & 5 & 4 & 3 & 5 & 4 & 3 \\
\hline $5 \mathbf{b}$ & 7 & 6 & 4 & 7 & 6 & 5 \\
\hline $5 c$ & 10 & 9 & 8 & 10 & 9 & 8 \\
\hline $5 d$ & 8 & 7 & 5 & 5 & 4 & 3 \\
\hline $5 e$ & 8 & 7 & 6 & 7 & 6 & 5 \\
\hline $5 \mathbf{f}$ & 7 & 6 & 5 & 7 & 6 & 5 \\
\hline $5 \mathrm{~g}$ & NA & NA & NA & NA & NA & NA \\
\hline $5 \mathrm{~h}$ & 6 & 5 & 4 & 6 & 5 & 4 \\
\hline Fluconazole & 16 & 16 & 15 & 18 & 17 & 15 \\
\hline
\end{tabular}

The results of antimicrobial screening revealed that some of the tested compounds showed moderate to good bacterial inhibition whereas all the compounds were exhibited moderate to weak fungal inhibition. As per structural discussion of these products studies and explained that the different substituent on the aromatic ring present in the synthesized compounds exhibited a significant influence on the biological activity. The presence of electron-withdrawing group on the aromatic ring increases the antibacterial activity of tested compounds compared to compounds having electron donating groups. Compounds $\mathbf{3 b}, \mathbf{3 d}, \mathbf{4 b}, \mathbf{4 d}, \mathbf{5 b}, \mathbf{5 d}$ and $\mathbf{5 c}$ have shown good zone of inhibition against $S$. aureus and $E$. coli with three different concentrations. The compounds such as 2-(4-chloro-phenyl)-3-(3-hydroxy4-(1H-benzo[d]imidazol-2-yl)phenyl)thiazolidin-4-one, 3-(3hydroxy-4-(1H-benzo[d]imidazol-2-yl)phenyl)-2-(4-nitrophenyl)thiazolidin-4-one and 4-((3-(1H-benz[d]imidazol-2-yl)6-(2-(4-chlorophenyl)-4-oxothiazolidin-3-yl)-2-hydroxyphenyl)diazenyl)benzene sulfonamide exhibited more potent antibacterial activity against four bacterial strains and the compound 3-(4-(1H-benzo[d]imidazol-2-yl)-3-hydroxyphenyl)-2-o-tolylthiazolidin-4-one was no activities against all bacterial and fungal strains. The tested compounds such as $\mathbf{4 b}$ and $\mathbf{5 b}$ were excellent inhibition activity against $S$. aureus, $E$. coli and $P$. aeruginosa. The compounds $\mathbf{3 a}, \mathbf{3 c}, \mathbf{3 f}, \mathbf{4 a}, \mathbf{4 c}$ and $\mathbf{5 a}$ were found to moderate to weak activities against all bacterial stains but all the synthesized Schiff's base and thiazolidinone derivatives products were ineffective against S. typhi. These results revealed the importance of benzimidazolyl and sulphomoyl substituted 4-thiazolidinone ring systems as basic structural constituents in the synthesized compounds. The substitution of chloro, nitro and sulphonamide group C-4 position of the phenyl ring and azosulphonamide bearing 4-thiazolidinone ring presence to be very important for antibacterial effect. Among all the antifungal tested synthesized compounds $\mathbf{4 b}$ and $\mathbf{5 c}$ were found to be moderate activity against fungal strains.

\section{Conclusion}

Present research work involves synthesis of benzimidazole bearing 4-thiazolidinone derivative to explore their antimicrobial activity. All the compounds were recrystallized, checked the purity by TLC and functionally interpreted by IR and ${ }^{1} \mathrm{H}$ NMR. In general from of the above results, it was concluded the compounds having chlorine, nitro and sulphonamide at C-4 position of the substituted phenyl which enhanced the activity due to presence electron withdrawing functional groups.

\section{REFERENCES}

1. M.G. Vigorita, R. Ottanà, F. Monforte, R. Maccari, A. Trovato, M.T. Monforte and M.F. Taviano, Bioorg. Med. Chem. Lett., 11, 2791 (2001).

2. C.V. Kavitha, Basappa, S.N. Swamy, K. Mantelingu, S. Doreswamy, M.A. Sridhar, J. Shashidhara Prasad and K.S. Rangappa, Bioorg. Med. Chem., 14, 2290 (2006).

3. G. Küçükgüzel, A. Kocatepe, E. De Clercq, F. Sahin and M. Güllüce, Eur. J. Med. Chem., 41, 353 (2006).

4. Z. Turgut, C. Yolacan, F. Aydogan, E. Bagdatli and N. Ocal, Molecules, 12, 2151 (2007).

5. G. Kucukguzel, E.E. Oruc, S. Rollas, F. Sahin and A. Ozbek, Eur. J. Med. Chem., 37, 197 (2002).

6. N. Karali, E. Illhan, A. Gürsoy and M. Kiraz, Farmaco, 53, 346 (1998).

7. N. Ergenc and G. Capan, Farmaco, 49, 133(1994).

8. L. Bukowski, M. Janowiec, Z. Zwolska-Kwiek and Z. Andrezejczyk, Pharmazie, 53, 373 (1998).

9. J.J. Bhatt, B.R. Shah, H.P. Shah, P.B. Trivedi, N.K. Undavia and N.C. Desai, Indian J. Chem., 33B, 189 (1994).

10. G.C. Look, J.R. Schullek, C.P. Holmes, J.P. Chinn, E.M. Gordon and M.A. Gallop, Bioorg. Med. Chem. Lett., 6, 707 (1996).

11. M.V. Diurno, O. Mazzoni, E. Piscopo, A. Calignano, F. Giordano and A. Bolognese, J. Med. Chem., 35, 2910 (1992).

12. A.R. Saundane, M. Yarlakatti, P. Walmik and V. Katkar, J. Chem. Sci., 124, 469 (2012).

13. Y. Suzuki, M. Akima and K. Tamura, Gen. Pharmacol., 32, 57 (1999).

14. A.G. Habeeb, P.N. Praveen Rao and E.E. Knaus, J. Med. Chem., 44, 3039 (2001).

15. J. Abhishek Kumar, V. Ankur, R. Veerasamy, K.K. Sushil and K.A. Ram, Bioorg. Med. Chem., 20, 3378 (2012).

16. I.M.A. Awad, A.A.M. Aly, A.M. Abdel-Alim, R.A. Abdel-Aal and S.H. Ahmed, J. Inorg. Biochem., 33, 77 (1998).

17. A.G. Macsumov, M. Ergashev and F.A. Normative, Pharm. Chem. J., 25 (1991).

18. A.A. Jarrahpour, M. Motamedifar, K. Pakshir, N. Hadi and M. Zare, Molecules, 9, 815 (2004).

19. S.M. Koshti, J.P. Sonar and D.H.I. More, Indian J. Chem., 47B, 329 (2008).

20. A.R. Tewari and A. Mishra, Indian J. Chem., 45B, 489 (2006). 